\title{
Entanglement, magnetic and quadrupole moments properties of the mixed spin Ising-Heisenberg diamond chain
}

\author{
V. S. Abgaryan ${ }^{1}$, N. S. Ananikian², L. N. Ananikyan², V. Hovhannisyan² \\ ${ }^{1}$ Bogoliubov Laboratory of Theoretical Physics, Joint Institute for Nuclear Research, \\ 141980 Dubna, Russia \\ 2 Alikhanyan National Science Laboratory, Alikhanian Br. 2, 0036 Yerevan, Armenia
}

\begin{abstract}
Thermal entanglement, magnetic and quadrupole moments properties of the mixed spin- $\frac{1}{2}$ and spin-1 Ising-Heisenberg model on a diamond chain are considered. Magnetization and quadrupole moment plateaus are observed for the antiferromagnetic couplings. Thermal negativity as a measure of quantum entanglement of the mixed spin system is calculated. Different behavior for the negativity is obtained for the various values of Heisenberg dipolar and quadrupole couplings. The intermediate plateau of the negativity has been observed at absence of the single-ion anisotropy and quadrupole interaction term. When dipolar and quadrupole couplings are equal there is a similar behavior of negativity and quadrupole moment.

Keywords: C. Ising-Heisenberg diamond chain; D. magnetization plateaus; D. quantum entanglement
\end{abstract}

\section{Introduction}

Quantum phase transitions (QPTs) [1], occurring at a zero temperature are triggered by changes of external parameters as a consequence of pure quantum fluctuations. These quantum transitions are typical for strongly correlated systems. It is known that the system wavefunction, in general, cannot be factorized into a direct product of subsystem states due to the nature of quantum entanglement. This phenomenon, that does not appear in the classical theory, is under great attention due to its importance in 
various aspects of quantum information science, ranging from discrete to continuous variable quantum computation and communication [2, 3, 4, 5]. Even more, being a correlational measure by nature, entanglement can be a crucial characteristic of QPTs [6, 7]. The studies of entangled chains, rings, molecules, Heisenberg models, and cluster states [8, 9, 10, 11], which are N-qubit systems, are focused on the two-qubit quantum correlations.

In the present paper we have chosen negativity [12] as a calculable measure of entanglement, to study quantum phase transitions and thermal entanglement in a mixed spin-(1/2-1) Ising-Heisenberg diamond chain. Thermal entanglement was detected by both experimental [13, 14, 15] and theoretical [16] observations at low dimensional spin systems, formed in compounds.

Models on diamond chains, exhibiting interesting quantum magnetic phenomena have recently been intensively studied theoretically $[17,18,19,20,21$, 22, 23, 24, 25, 26, 27]. The interest to this type of system has been increased since experimental work of Kikuchi and co-workers [28, 29], where natural mineral azurite $\left(\mathrm{Cu}_{3}\left(\mathrm{CO}_{3}\right)_{2}(\mathrm{OH})_{2}\right)$ has been recognized as an appropriate candidate for diamond chain compound. There have been proposed different types of theoretical Heisenberg models to explain the experimental measurements of magnetization plateau and the double peak behavior in the natural mineral azurite (the density-matrix and transfer-matrix renormalizationgroup techniques, density functional theory, high-temperature expansion, variation mean-field-like treatment, based on the Gibbs-Bogoliubov inequality) [30, 31, 32, 33]. Magnetization plateaus and the multiple peak structure of the specific heat have also been observed on an Ising-Hubbard diamond chain [34].

The paper is organized as follows. In Section 2 we introduce the exactly solvable mixed spin-1/2 and spin-1 Ising-Heisenberg diamond chain with its magnetic and quadrupole moments properties. Results for thermal negativity with and without quadrupolar interactions and single-ion anisotropy are presented in Section 3. Finally, conclusions and future prospects are briefly mentioned in Section 4 .

\section{Model and thermodynamics}

We consider the spin- $\frac{1}{2}$ and spin-1 Ising-Heisenberg model on a diamond chain with quadrupole couplings and single-ion anisotropy (longitudinal crystal field) in the presence of the external magnetic field. The diamond chain 
(Fig. 1) is a quasi one-dimensional system, consisting of the nodal spins, alternating with vertical dimers. The Hamiltonian of the model may be written in the following form:

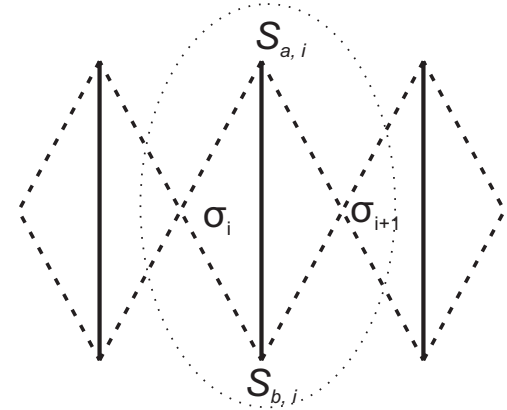

Figure 1: A schematic representation of the mixed spin diamond chain. Dashed (solid) lines correspond to Ising (Heisneberg)-type interactions.

$$
\begin{aligned}
H & =\sum_{i=1}^{N} H_{i}, \\
H_{i} & =J_{0}\left(\vec{S}_{a, i} \vec{S}_{b, i}\right)+J_{1}\left(\sigma_{i}^{z}+\sigma_{i+1}^{z}\right)\left(S_{a, i}^{z}+S_{b, i}^{z}\right)+K\left(\vec{S}_{a, i} \vec{S}_{b, i}\right)^{2} \\
& +D\left(\left(S_{a, i}^{z}\right)^{2}+\left(S_{b, i}^{z}\right)^{2}\right)-h\left(\frac{\sigma_{i}^{z}}{2}+S_{a, i}^{z}+\frac{\sigma_{i+1}^{z}}{2}+S_{b, i}^{z}\right) .
\end{aligned}
$$

Here $\sigma_{i}^{z}$ is Pauli $z$ matrix, $S_{a, i}^{\alpha}$ and $S_{b, i}^{\alpha}(\alpha=x, y, z)$ are components of spin-1 operators. $\quad J_{0}$ and $K$ are coefficients of dipolar and quadrupolar interactions within spin-1 dimer, respectively. $J_{1}$ stands for Ising-type interactions between nodal spin-1/2 Ising and spin-1 Heisenberg sites. Coefficient $D$ stands for the single-ion anisotropy (the longitudinal crystal field) and the last term $(h)$ is the contribution of the external magnetic filed. At a special point $J_{0}=K$ the system undergoes qualitative changes, namely the operator $\sum_{i=1}^{N}\left(\left(S_{a, i}^{z}\right)^{2}+\left(S_{b, i}^{z}\right)^{2}\right)$ commutes with the total Hamiltonian and a new order parameter (quadrupole moment) can be used to describe the properties of the system.

The important part of our further calculations is based on the commutation relation between different block Hamiltonians $\left[\mathcal{H}_{i}, \mathcal{H}_{j}\right]=0$ which means that the blocks of diamond chain are separable. So in order to gain the entanglement of the whole chain it is sufficient to calculate entanglement of single block. 
For precise solution of the model we have to consider only the problem of the single block Hamiltonian. One may check that within each block the Heisenberg part commutes with the Ising one. This allows us to trace out Ising spins from the very beginning and consider only the quantum problem of spin-1 dimer (entanglement properties of this model are considered in [35]). Formally, this means that we can simply replace the $\sigma_{i}^{z}$ Pauli matrixes by $\sigma_{i}= \pm 1 / 2$ numbers. Hence, nine eigenvalues $\left(\lambda_{n}\left(\sigma_{i}, \sigma_{i+1}\right), n=1, . ., 9\right)$ of the i-th block Hamiltonian can analytically be found (the corresponding eigenvectors are also given in [35]):

$$
\begin{aligned}
& \lambda_{1,2}=J_{0}+2 D+K-\left(\frac{h}{2} \pm 2 J_{1}\right)\left(\sigma_{i}+\sigma_{i+1}\right) \pm 2 h, \\
& \lambda_{3,4}=J_{0}+D+K-\left(\frac{h}{2} \pm J_{1}\right)\left(\sigma_{i}+\sigma_{i+1}\right) \pm h, \\
& \lambda_{5,6}=-J_{0}+D+K-\left(\frac{h}{2} \pm J_{1}\right)\left(\sigma_{i}+\sigma_{i+1}\right) \pm h, \\
& \lambda_{7}=-J_{0}+2 D+K-\frac{1}{2} h\left(\sigma_{i}+\sigma_{i+1}\right), \\
& \lambda_{8,9}=\frac{1}{2}\left(-J_{0}+2 D+5 K-h\left(\sigma_{i}+\sigma_{i+1}\right) \pm v\right),
\end{aligned}
$$

where by $v$ we denoted:

$$
v=\sqrt{\left(-2 D+J_{0}-K\right)^{2}+8\left(J_{0}-K\right)^{2}} .
$$

We are going to solve the model by direct transfer matrix method. For this purpose let us write the partition function of the model in this form:

$$
Z=\sum_{\sigma_{i}} \prod_{i=1}^{N} \operatorname{Tr}_{i} \mathrm{e}^{-\beta \mathcal{H}_{\mathrm{i}}},
$$

where $\beta=\left(k_{B} T\right)^{-1}, k_{B}$ is Boltzmann's constant, $T$ is the absolute temperature. After performing a trace over the spin-1 Heisenberg dimers, one can rewrite the partition function into the following form

$$
Z=\sum_{\sigma_{i}} \prod_{i=1}^{N} T_{\sigma_{i}, \sigma_{i+1}}=\operatorname{Tr} T^{N}
$$


here, $T_{\sigma_{i}, \sigma_{i+1}}$ is the standard $2 \times 2$ transfer matrix:

$$
T_{\sigma_{i}, \sigma_{i+1}}=\left(\begin{array}{cc}
T_{+,+} & T_{+,-} \\
T_{-,+} & T_{-,-}
\end{array}\right)
$$

where, \pm denote two spin states of the Ising spins $\sigma_{i}= \pm \frac{1}{2}$. The elements of the transfer matrix are defined through eigenvalues (2) as

$$
T_{\sigma_{i}, \sigma_{i+1}}=\operatorname{Tr}_{i} \mathrm{e}^{-\beta \mathcal{H}_{\mathrm{i}}}=\sum_{\mathrm{n}=1}^{9} \mathrm{e}^{-\beta \lambda_{\mathrm{n}}\left(\sigma_{\mathrm{i}}, \sigma_{\mathrm{i}+1}\right)} .
$$

After this, the total partition function takes the form similar to the partition function of a one dimensional chain, with two-value classical variables on each site:

$$
Z=\Lambda_{1}^{N}+\Lambda_{2}^{N}
$$

where $\Lambda_{1,2}$ are the eigenvalues of transfer matrix (6). Then we take into account that in the thermodynamic limit it is sufficient to consider only the largest eigenvalue to calculate the partition function:

$$
f=-\frac{1}{\beta} \ln \frac{1}{2}\left(T_{+,+}+T_{-,-}+\sqrt{\left(T_{+,+}-T_{-,-}\right)^{2}+4 T_{+,-}^{2}}\right) .
$$

The basic order parameter for this model is the per block magnetization, defined as

$$
m=-\left(\frac{\partial f}{\partial h}\right)_{T, D} .
$$

As it is already mentioned above there is a special parametrization $J_{0}=K$ with another order parameter (quadrupole moment)

$$
q=\left(\frac{\partial f}{\partial D}\right)_{J_{0}=K, T, D} .
$$

General features of magnetic and quadrupole moments behavior are presented in Fig. 2. First of all, let us turn our attention to the behavior of magnetization. Figure 2(a) shows field dependence of the magnetization at low temperature for the different values of exchange couplings $J_{0}$ and $J_{1}\left(J_{0}=1, J_{1}=1\right.$ solid curve, $J_{0}=1, J_{1}=-1$ dot-dashed curve, 
$J_{0}=-1, J_{1}=1$ dashed curve). All calculations in this case have been carried out when quadrupolar coupling and single-ion anisotropy are absent. The phase diagram has shown to be rather rich, demonstrating large variety of ground states [18].

The most general magnetization curve when both exchange parameters are antiferromagnetic (solid line) shows the existence of two intermediate plateaus at $1 / 5$ and $3 / 5$ of the saturation magnetization. Plateau at $1 / 5$ corresponds to frustrated state with dimer magnetization $\left\langle S^{z}\right\rangle=0$ (eigenvalues $\left.\lambda_{8,9}\right)$ or state with dimer magnetization $\left\langle S^{z}\right\rangle=1$, depending on the Ising spins orientation. Magnetization plateau at $3 / 5$ corresponds to the dimer magnetization $\left\langle S^{z}\right\rangle=1$, (more precisely to the part of block-Hamiltonian with eigenvalues $\left.\lambda_{5,6}\right)$. The magnetization reaches its saturation value at higher magnetic fields $(h>4)$, thus the dimer magnetization of the ground state is $\left\langle S^{z}\right\rangle=2$.

If the exchange parameters have different sign (dot-dashed and dashed curves Fig. 2(a)), then, we may observe only one intermediate plateau at 3/5 with different values of dimer magnetization. In the case when Heisenberg interaction is ferromagnetic (dot-dashed curve) dimer magnetization reaches its maximum value and remains the same for all positive values of magnetic field. The appearance of plateau at $3 / 5$ is a consequence of stepping from one set of values of classical variables (Ising spins are parallel to the dimer spins) into another (Ising spins are antiparallel to them). Finally, when Heisenberg interaction is antiferromagnetic (dashed curve) transition from plateau at $3 / 5$ to the saturation value takes place between states of the dimer (from the state $\left\langle S^{z}\right\rangle=1$ to a state $\left\langle S^{z}\right\rangle=2$ ). The transition between sets of values of classical variables in particular means that entanglement of system does not change. There are two order parameters (magnetic and quadrupole moments), when $K=J_{0}$. Figure 2(b) shows field dependence of the magnetization, when quadrupolar coupling and single-ion anisotropy are presented. For all cases, one may observe the existence of the magnetization plateau at $1 / 5$ in low values of the external magnetic field. In the case when Heisenberg interaction is antiferromagnetic (solid and dot-dashed curves) there is an intermediate plateau at $3 / 5$ with the same value of the dimer magnetization $\left\langle S^{z}\right\rangle=1$.

In Fig. 2 (c) and (d) we plotted low-temperature quadrupole moment curves for different values of parameters and magnetic field. One observes only one intermediate plateau at $1 / 2$ when Heisenberg interaction is antiferromagnetic (solid and dot-dashed curves). The qualitative comparison 
between quadrupole moment and thermal entanglement will be given in the next Section.

(a)

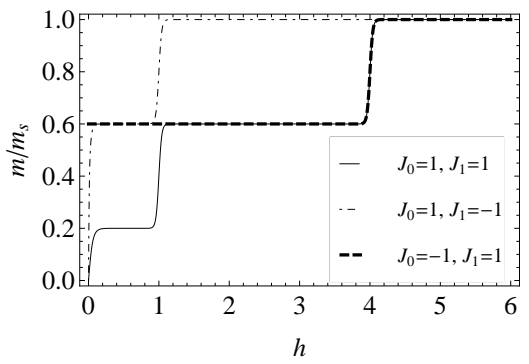

(c)

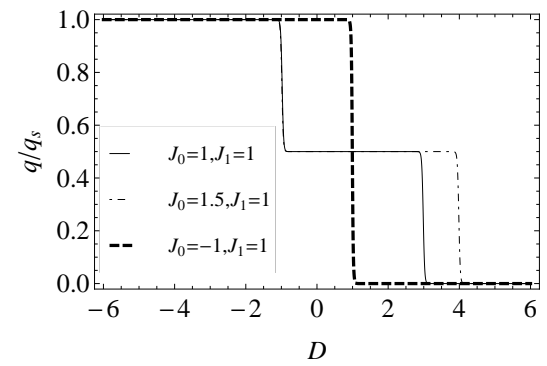

(b)

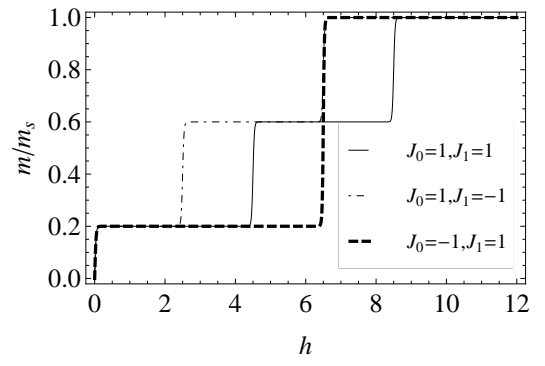

(d)

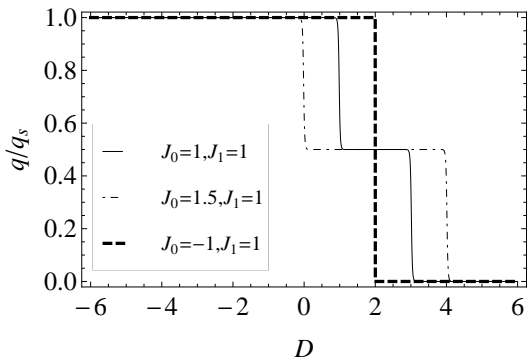

Figure 2: The magnetic-field dependence of the total magnetization at low temperature ( $T=0.02$ ) for different values of exchange parameters. (a) $K=D=0$, (b) $J_{0}=K$ and $D=5.5$. The single-ion anisotropy dependence of the total quadrupole moment for different values of exchange parameters and magnetic field (c) $h=0$, (d) $h=2$.

\section{Thermal negativity}

In this Section, we use negativity as a computable measure of pairwise entanglement [12], which for the density matrix $\rho$ is defined as:

$$
N e(\rho) \equiv \frac{\left\|\rho^{T_{1}}\right\|_{1}-1}{2},
$$

where $\left\|\rho^{T_{1}}\right\|$ is the trace norm of the partial transposed $\rho^{T_{1}}$ of a bipartite density matrix $\rho$. 
At a thermal equilibrium the density matrix of the $i$-th block is given as

$$
\rho(T)=\sum_{j=1}^{36} \frac{e^{-\frac{E_{j}}{k_{B} T}}}{Z_{\text {block }}}\left|\psi_{j}\right\rangle\left\langle\psi_{j}\right|,
$$

where $E_{j}$ 's are eigenvalues of the block Hamiltonian $H_{i}$ corresponding to the states $\left|\psi_{j}\right\rangle$. Now let us consider negativity properties of the mixed spin Ising-Heisenberg diamond chain with and without quadrupolar coupling and single-ion anisotropy.

\subsection{Chain negativity when $K=0, D=0$}

For understanding the behavior of entanglement and QPT features we plot in Fig. 3 a typical behavior of the negativity when quadrupolar coupling and single-ion anisotropy are absent. Figure 3 (a) shows magnetic field and dipolar parameter dependence of the negativity with antiferromagnetic Ising interaction $\left(J_{1}=1\right)$. As one finds, for $0.5<J_{0}<1$ only a transition between non-entangled $\left\langle S^{z}\right\rangle=2$, and partially entangled $\left\langle S^{z}\right\rangle=1$ states occurs, when crossing $|h|=2 J_{0}-1$ line. This transition corresponds to the magnetization jump from a plateau at $3 / 5$ to $1 / 5$. Increasing the Heisenberg coupling $\left(1<J_{0}<1.5\right)$, we have a transition between non-entangled and fully entangled $\left(\left\langle S^{z}\right\rangle=0\right)$ states that cross at $|h|=3 J_{0}-2$ line. Furthermore, for stronger Heisenberg interaction coupling $\left(J_{0}>1.5\right)$ the following scenario takes place. Decreasing the absolute value of the magnetic field and crossing the line $|h|=2 J_{0}+1$, we arrive from a non-entangled state to a partially entangled one. Finally, for even weaker magnetic fields a transition from partially entangled state to fully entangled one occurs at the line $|h|=2 J_{0}+2$. Negativity shows a similar behavior when Ising interaction is a ferromagnetic one (Fig. 3(b)).

As for the thermal behavior of the negativity we note that it decreases monotonically with the temperature growth, due to decoherence effects. Particularly, for $J_{0}= \pm J_{1}$ and $h=0$ the critical temperature of entanglement vanishing is $T_{c}=\frac{J_{0}}{\ln 2.98} \approx 0.915 J_{0}$, while for $J_{0} \neq \pm J_{1}$ and $h=0$, $T_{c}=\frac{J_{0}-J_{1}}{\ln 2.96} \approx 0.921\left(J_{0}-J_{1}\right)$.

\subsection{Negativity in special case $K=J_{0}$}

Now let us turn our attention to the properties of the negativity with presence of the quadrupolar coupling and single-ion anisotropy. The typical magnetic field and dipolar parameter dependence of the negativity is shown 
(a)

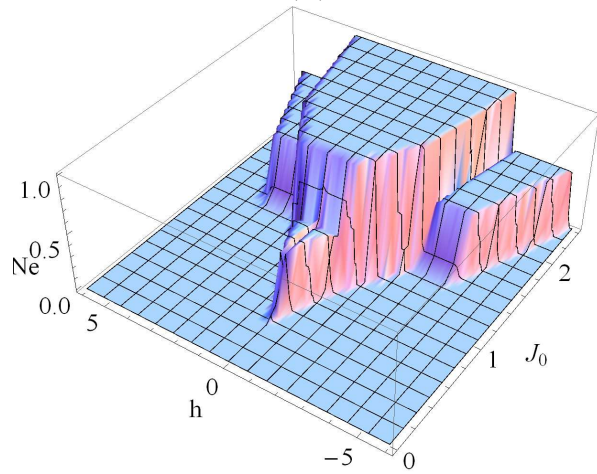

(b)

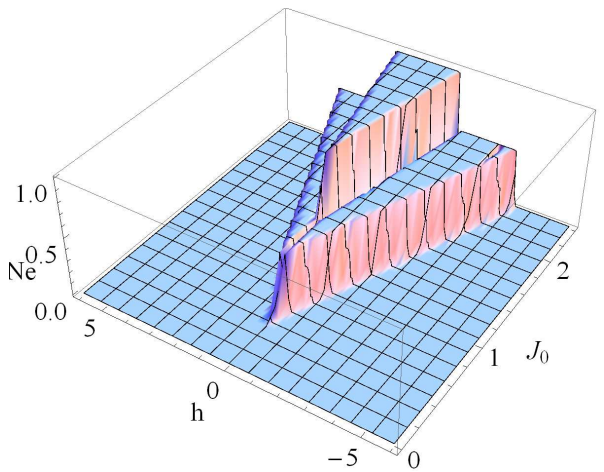

Figure 3: Negativity against Heisenberg coupling parameter $J_{0}$ and magnetic field $h$ for (a) antiferromagnetic Ising interaction $J_{1}=1$ (b) ferromagnetic Ising interaction $J_{1}=-1$, at low temperature $(T=0.02)$ when $K=0, D=0$.

in Fig. [4 for two qualitatively different values of the single-ion anisotropy. One can note that $K=J_{0}$ condition brings to the disappearance of the fully entangled states, which means that the saturation value of the thermal negativity is 0.5 .

(a)

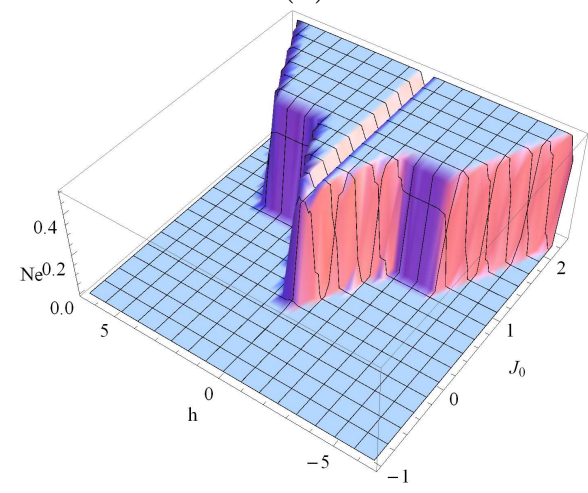

(b)

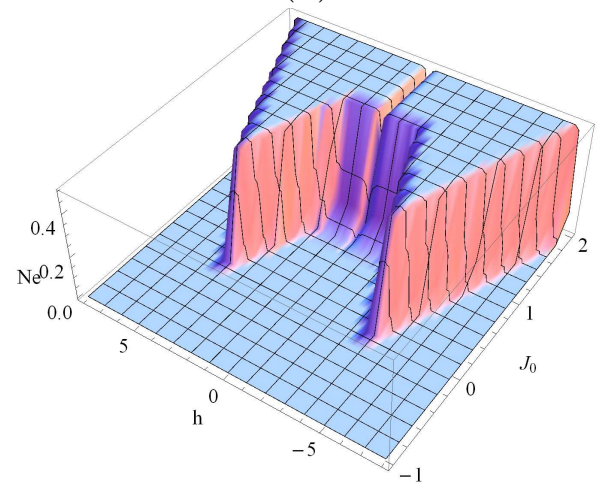

Figure 4: Negativity against magnetic field and parameter $J_{0}=K$ for the fixed value of the exchange parameter $J_{1}=1$ and absolute temperature $T=0.1$ and for the different values of the single-ion anisotropy (a) $D=1$, (b) $D=3$. 
(a)

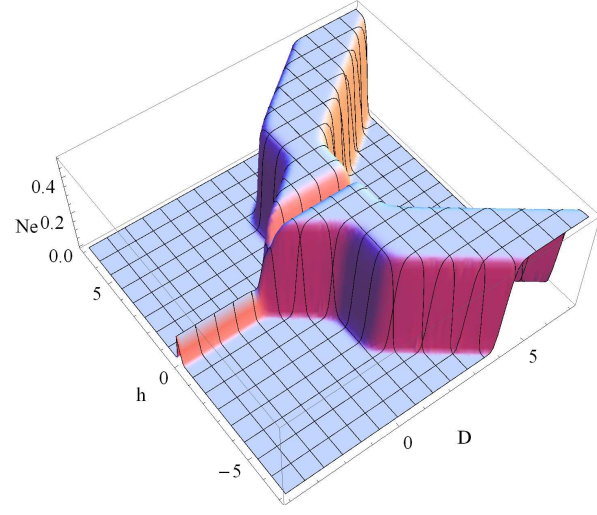

(b)

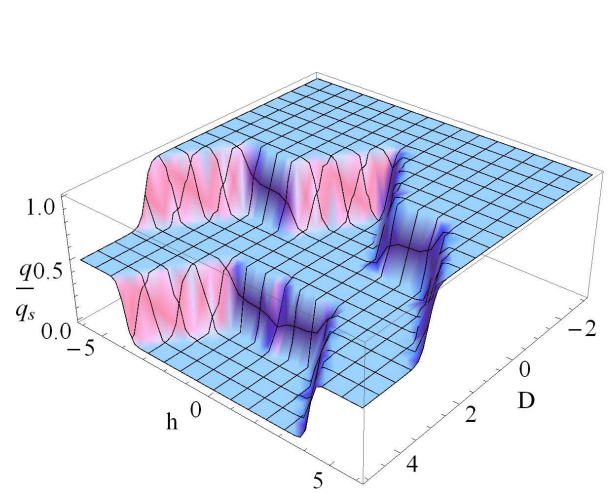

Figure 5: Negativity via magnetic field $h$ and single-ion anisotropy $D$ when $J_{0}=J_{1}=$ $K=1$ and $T=0.1$, (a) quadrupole moment for same values of parameters with respect to its saturation value (b).

Finally, let us compare properties of the thermal negativity and quadrupole moment of the system. Figure 5 (a) shows magnetic field and single-ion anisotropy dependence of the negativity for the fixed value of the exchange parameters $J_{0}=J_{1}=K=1$ and absolute temperature $T=0.1$. As one can see, there are only two different regimes of the negativity. Plateau at zero corresponds to non-entangled regime, while plateau at one-half corresponds to partially entangled one. Fig. 沾 (b) shows a three-dimensional plot of the quadrupole moment as a function of the magnetic field and single-ion anisotropy for the same values of the exchange parameters and absolute temperature. One may differ three different regions of the quadrupole moment. The first one is the region with zero quadrupole moment, where the system is non-entangled with dimer magnetization $\left\langle S^{z}\right\rangle=0$. Then, there is the region, where quadrupole moment has an intermediate plateau at one-half of the saturation value. At this region the system is partially entangled with the maximal value of the negativity. Afterwards, there comes a region with maximal value of the quadrupole moment $q / q_{s}=1$, where the system is separable, therefore non-entangled (with the exception of the line $h=0$ ). 


\section{Conclusion}

In the present work, the mixed spin- $1 / 2$ and spin-1 Ising Heisenberg model on a diamond chain has been exactly solved by the transfer matrix method. In particular, we have studied the magnetic and quadrupole moment properties of the system with and without quadrupolar coupling and single-ion anisotropy. Particulary, we have shown the existence of two intermediate magnetization plateaus at one-fifth and three-fifth of the saturation magnetization. One may also observe the existence of the plateau on the quadrupole moment curve in the antiferromagnetic case. Using thermal negativity as a measure of entanglement, we observe strong correlations between magnetization, quadrupole moment and negativity. Entanglement properties of the model strongly depend on the exchange parameters. In particular, we obtain two or three different entangled regimes of the system, which strongly depend on the presence of the quadrupolar coupling and single-ion anisotropy.

\section{Acknowledgments}

The authors would like to thank L.A. Chakhmakhchyan for useful discussion. N A acknowledges financial support by the MC-IRSES No. 612707 (DIONICOS) under FP7-PEOPLE-2013 and research project No. SCS 131C137 grants. V H would like to acknowledge support from the NFSAT, the Young Scientists Support Program (YSSP), Youth Foundation of Armenia (AYF) and CRDF Global under grant YSSP-13-02.

\section{References}

\section{References}

[1] S. Sachdev Quantum Phase Transitions. Cambridge University Press, Cambridge, England (1999).

[2] M. A. Nielsen and I. L. Chuang, Quantum Computation and Quantum Information. Cambridge University Press, Cambridge, England (2000).

[3] L. Chakhmakhchyan, S. Guérin, J. Nunn, and A. Datta, Phys. Rev. A 88 (2013) 042312. 
[4] R. Ursin, F. Tiefenbacher, T. Schmitt-Manderbach, H. Weier, T. Scheidl, M. Lindenthal, B. Blauensteiner, T. Jennewein, J. Perdigues, P. Trojek, B. Ömer, M. Furst, M. Meyenburg, J. Rarity, Z. Sodnik, C. Barbieri,H.Weinfurter, and A. Zeilinger, Nat. Phys. 3 (2007) 481.

[5] A. Avella, G. Brida, I. P. Degiovanni, M. Genovese, M. Gramegna, and P. Traina, Phys. Rev. A 82 (2010) 062309.

[6] L. Amico, R. Fazio, A. Osterloh and V. Vedral, Rev. Mod. Phys. 80 (2008) 517.

[7] R. Horodecki P. Horodecki, M. Horodecki and K. Horodecki, Rev. Mod. Phys. 81 (2009) 865.

[8] W. K. Wootters, Contemporary Mathematics 305 (2002) 299.

[9] F. Benatti, B. C. Hiesmayr, H. Narnhofer, Eur.Phys. Lett. 72 (1) (2005) 28.

[10] K. M. O’Connor and W. K. Wootters, Phys. Rev. A 63 (2001) 052302.

[11] H. J. Briegel and R. Raussendorf, Phys. Rev. Lett. 86 (2001) 910.

[12] G. Vidal, R. F. Werner, Phys. Rev. A 65 (2002) 032314.

[13] A. M. Souza, M. S. Reis, D. O. Soares-Pinto, I. S. Oliveira and R. S. Sarthour, Phys. Rev. B 77 (2008) 104402.

[14] T. G. Rappoport, L. Ghivelder, J. C. Fernandes, R. B. Guimares and M. A. Continentino, Phys. Rev. B 75 (2007) 054422.

[15] A. M. Souza, D. O. Soares-Pinto, R. S. Sarthour, I. S. Oliveira, M. S. Reis, P. Brando, and A. M. dos Santos, Phys. Rev. B 79 (2009) 054408.

[16] N. S. Ananikian, L. N. Ananikyan, L. A. Chakhmakhchyan and A. N. Kocharian, J. Phys. A: Math. Theor. 44 (2011) 025001.

[17] K. Takano, K. Kubo and H. Sakamoto, J. Phys.: Condens. Matter 8 (1996) 6405.

[18] L. Canova, J. Strečka and M. Jaščur, J. Phys.: Condens. Matter 18 (2006) 4967. 
[19] N. B. Ivanov, J. Richter and J. Schulenburg, Phys. Rev. B 79 (2009) 104412.

[20] K. Hida, K. Takano and H. Suzuki, J. Phys. Soc. Japan 79 (2010) 044702.

[21] J. Schulenburg, A.Honecker, J.Schnack, J.Richter and H-J. Schmidt, Phys. Rev. Lett. 88 (2002) 167207.

[22] O. Rojas, M. Rojas, N. S. Ananikian and S. M. de Souza, Phys. Rev. A 86 (2012) 042330.

[23] N. S. Ananikian, V. V. Hovhannisyan, Physica A 392 (2013) 2375.

[24] L. Canova, J. Strečka, T. Lucivjansky, Condens. Matter Phys. 12 (2009) 353.

[25] N.S. Ananikian, J. Strečka, V. Hovhannisyan, Solid State Commun. 194 (2014) 48.

[26] O. Rojas, S. M. de Souza, V. Ohanyan, and M. Khurshudyan, Phys. Rev. B 83 (2011) 094430.

[27] N. S. Ananikian, L. N. Ananikyan, L. A. Chakhmakhchyan, O. Rojas, J. Phys.: Condens. Matter 24 (2012) 256001.

[28] H. Kikuchi, Y. Fujii, M. Chiba, S. Mitsudo, T. Idehara, T. Tonegawa, K. Okamoto, T. Sakai, T. Kuwai and H. Ohta, Phys. Rev. Lett. 94 (2005) 227201.

[29] H. Jeschke, I. Opahle, H. Kandpal, R. Valent, H. Das, T. Saha-Dasgupta, O. Janson, H. Rosner, A. Brhl, B. Wolf, M. Lang, J. Richter, S. Hu, X. Wang, R. Peters, T. Pruschke, A. Honecker, Phys. Rev. Lett. 106 (2011) 217201.

[30] A. Honecker and A. Lauchli, Phys. Rev. B 63 (2001) 174407.

[31] B. Gu and G. Su, Phys. Rev. B 75 (2007) 174437.

[32] J. Kang, C. Lee, R. K. Kremer and M-H. Whangbo, J. Phys.: Condens. Matter 21 (2009) 392201.

[33] N. Ananikian, H. Lazaryan, and M. Nalbandyan, Eur. Phys. J. B 85 (2012) 223. 
[34] M. Nalbandyan, H. Lazaryan, O.Rojas, S. M. de Souza, N. S. Ananikian, J. Phys. Soc. Jpn. 83 (2014) 074001.

[35] V. S. Abgaryan, N. S. Ananikian, L. N. Ananikyan and A. N. Kocharyan, Phys. Scr. 83, (2011) 055702. 